\title{
Effects of Nutritional Status on 6-Month Outcome of Hip Fractures in Elderly Patients
}

\author{
Ka Ying Doris Miu, MBBS, MRCP, MPH, Pui Shan Lam, MBBS, MRCP \\ Department of Rehabilitation and Extended Care, Wong Tai Sin Hospital, Hong Kong
}

\begin{abstract}
Objective To identify the prevalence of malnutrition in elderly hip fracture and to investigate the relationship between hip fracture patients and malnutrition on functional recovery and mortality.

Methods All hip fracture patients age $>65$ years admitted to a rehabilitation unit were recruited from July 2015 to June 2016. Nutritional status was assessed by Mini-Nutritional Assessment Short-Form (MNA-SF) within 72 hours of admission. Patients were reassessed at 6 months for functional status and place of residence. Length of hospital stay, in-patient mortality rate, and 6-month mortality rate were also recorded.

Results There were 218 patients recruited. The mean age was $83.5 \pm 7.5$ years. According the MNA-SF, 46 (21.1\%) were well nourished, 115 (52.6\%) were at risk of malnutrition, and 57 (26.1\%) were malnourished. Malnourished individuals were significantly older, had lower Mini-Mental State Examination score and albumin level, were functionally more dependent and were more likely to reside in elderly care facility. A higher proportion of elderly care residents were at-risk or were malnourished on admission, discharge and at 6 months. Functional recovery was slower in the malnourished group. In-patient mortality was higher in malnourished individuals compared to those at risk of malnourishment and well-nourished individuals.

Conclusion The prevalence of malnutrition is high and is associated with poor functional recovery and elderly care placement. Residents of elderly care facilities are especially at risk due a higher prevalence of malnourishment. Health authorities are encouraged to evaluate the dietetic component in elderly care facilities and initiate nutrition supplementation in their planning of healthcare resources.
\end{abstract}

Keywords Hip fractures, Nutritional status, Outcome

\section{INTRODUCTION}

Hip fracture incidence is increasing among the elderly and is associated with increase in mortality, disability and dependency [1,2]. Functional recovery after hip fracture is an important health issue. Resumption of prefracture functional status is impaired 1 year after hip fracture in $25 \%-60 \%$ of older people [3].

Malnutrition in the elderly is an important global health problem that is associated with higher morbidity, mortal-

Received April 6, 2017; Accepted May 29, 2017

Corresponding author: Ka Ying Doris Miu

Department of Rehabilitation and Extended Care, Wong Tai Sin Hospital, 124 Shatin Pass Road 852, Hong Kong. Tel: +852-3517-3668, Fax: +852-35173855, E-mail: miuky@ha.org.hk

ORCID: Ka Ying Doris Miu (http://orcid.org/0000-0003-2923-4882); Pui Shan Lam (http://orcid.org/0000-0002-4340-5126).

@ This is an open-access article distributed under the terms of the Creative Commons Attribution Non-Commercial License (http://creativecommons.org/ licenses/by-nc/4.0) which permits unrestricted noncommercial use, distribution, and reproduction in any medium, provided the original work is properly cited. Copyright $\odot 2017$ by Korean Academy of Rehabilitation Medicine 
ity and lower quality of life. Malnutrition is prevalent in patients with various medical conditions including stroke and chronic respiratory failure, and in surgical patients [4]. Aging is also associated with physical, mental, social and environmental changes that can contribute to the risk of malnutrition [5].

Malnutrition is a broad term that is difficult to investigate. There is no universally accepted definition of malnutrition and the definition varies depending on the institution, discipline and culture. Malnutrition and under-nutrition are often used interchangeably [6] Under-nutrition is defined as a state of energy, protein or specific nutritional deficiency [7]. Protein is an important structural component and low protein intake may compromise the structure and strength of bone and lead to hip fracture $[8,9]$. Nutritional status in elderly patients with fracture can deteriorate during hospital stay [10] and protein energy malnutrition is a poor prognostic factor for functional recovery in hip surgery patients [10]. Energy expenditure and protein requirement are often elevated after a trauma from hip fracture and the following surgical repair. As a result, nutritional deficiency may be further exacerbated [11].

Protein energy malnutrition following acute hip fracture is associated with increased morbidity, mortality and healthcare costs [12]. In one study, up to $58 \%$ of patients with hip fracture were malnourished on admission or developed serious nutritional deficits while hospitalized, compared with only $2 \%$ of community dwelling individuals [10]. Malnutrition can be further aggravated by long delay for surgery, pain, nausea and vomiting [13]. The catabolic effect of surgery together with malnutrition leads to muscle wasting, which in turn will impair rehabilitation, prolong the length of hospitalization and result in poor clinical outcome and increased mortality $[5,10,14]$. Early identification and prompt treatment of malnutrition can help to decrease mortality and morbidity in elderly hip fracture patients.

Protein energy malnutrition screening and perioperative nutritional treatment have been recommended [15]. However, local available data is lacking. Moreover, data are scant concerning the nutritional status of elderly hip fracture patients. Furthermore, the prevalence of malnutrition in hip fracture patients is variable. Depending on the criteria used, the reported malnutrition rate varies from $6 \%-78 \%$ [16]. There is lack of universal consensus regarding the best measure for protein energy malnutrition diagnosis and documentation [17]. Commonly used markers for malnutrition in hip fracture studies include albumin level, body mass index (BMI), Mini Nutritional Assessment (MNA) or its short form (MNA-SF) [18]. However, all these measures are not routinely undertaken for hip fracture patients. So, the impact of malnutrition on patients and healthcare outcomes is likely under-recognized.

The aim of this study is to identify the prevalence of malnutrition in hip fracture elderly patients and to investigate the relationship between hip fracture elderly patients and malnutrition on functional recovery and 6-month mortality.

\section{MATERIALS AND METHODS}

Elderly ( $>65$ years of age) hip fracture patients admitted to our rehabilitation unit were recruited. Exclusion criteria were serious hearing problem, lack of ability to communicate, admittance for terminal illness with a limited life expectancy ( $<6$ months) and admittance for pathological fracture. All subjects provided consent for participation in the study. The next of kin or legal guardian provided consent for mentally incapable patients. Each patient was assessed within 72 hours of admission. Preoperative blood parameters including albumin and hemoglobin level were recorded in the acute orthopedic ward. Basic demographic data included age, sex and place of residence were collected. Medical comorbidities measured by Charlson Comorbidity Index (CCI), cognition measured by Mini-Mental State Examination (MMSE), type of surgery, interval between emergency admission and surgery and fracture type were also recorded. Nutritional status was assessed by MNA-SF [18]. The MNA-SF is a validated, sensitive, reliable and quick screening tool used in the elderly. It comprises six questions from the original MNA with a maximum score of 14. Items include a decline in food intake in the past 3 months due to loss of appetite or eating difficulty, weight loss during the past 3 months, neuropsychiatric problems (depression or dementia) and BMI or calf circumference. For those unable to respond to the questionnaire, information was obtained from their surrogate carers. Patients were classified as normal nutritional status if the score was 12-14, at risk of malnutrition for scores of 8-11, and 
malnourished for scores $<7$. Body weight was measured by chair scales using the Detecto 6475 device (Detecto, Webb City, MO, USA). Since all the patients had a hip fracture, height was measured using the half arm span, which is the distance from the midline at the sternal notch to the tip of the middle finger [19]. Height is then calculated by doubling the half arm span to the nearest $\mathrm{cm}$. Calf circumference was measured at the widest part of the calf when the patient was sitting with the nonfracture leg hanging loosely.

Pre-fracture functional status was categorized as fully independent, walk with aids or fully dependent. The Modified Barthel Index (MBI) was used to assess physical function. Discharge destination and length of stay in the rehabilitation unit were recorded. Patients were contacted by phone at 6 months post discharge to evaluate their functional status by MBI and place of residence. Mortality was recorded from the territory wide electronic record from the local health authority. Subjects that were classified as having malnourishment were seen by dietitian for nutritional supplement during their period of stay in hospital. This study is approved by the local hospital research ethics committee.

\section{Statistical analyses}

Between groups comparison for continuous data was performed by ANOVA and chi-square test for categorical data. Patients were classified into three groups according to the score of MNA-SF: normal, at risk of malnutrition, and malnourished. The association between nutritional status and functional recovery was assessed by ANOVA. Outcome on functional status at 3 months and 6 months with the nutritional group was analyzed by repeated measures ANOVA. In-patients and 6-month mortality were calculated and logistic regression was done after adjusting for factors that were significant in the univariate analysis. Odds ratio (OR) and $95 \%$ confidence interval (CI) were reported. A p-value $<0.05$ was considered statistically significant.

\section{RESULTS}

A total of 218 patients were recruited from July 2015 to June 2016. They comprised 144 (66.1\%) females and 74 (33.9\%) males. The mean age was $83.5 \pm 7.5$ years. The median CCI was 2 and MMSE was 17. Concerning the pre-fracture functional state, $84(38.5 \%)$ were indepen-

Table 1. Clinical and functional status by nutritional group

\begin{tabular}{|c|c|c|c|c|}
\hline & $\begin{array}{l}\text { Well nourished } \\
\qquad(n=46)\end{array}$ & $\begin{array}{c}\text { At risk } \\
(n=115)\end{array}$ & $\begin{array}{l}\text { Malnourished } \\
\quad(n=57)\end{array}$ & p-value \\
\hline Age (yr) & $80.2 \pm 7.6$ & $83.7 \pm 7.4$ & $85.7 \pm 6.9$ & 0.001 \\
\hline Sex, female $(\%)$ & 56.5 & 66.9 & 71.9 & 0.249 \\
\hline CCI (median) & 1 & 2 & 2 & 0.091 \\
\hline MMSE (median) & 23.5 & 18 & 11.35 & $<0.001$ \\
\hline Albumin (g/dL) & $34.8 \pm 5.1$ & $33.5 \pm 5.6$ & $31.5 \pm 6.9$ & 0.014 \\
\hline Hemoglobin $(\mathrm{g} / \mathrm{dL})$ & $11.9 \pm 1.9$ & $11.3 \pm 2.7$ & $10.9 \pm 3.7$ & 0.181 \\
\hline \multicolumn{5}{|l|}{ Functional status (\%) } \\
\hline Independent & 54.3 & 55.6 & 22.8 & \\
\hline Walk with aids & 45.7 & 55.7 & 65 & \\
\hline Chair/bed bound & 0 & 4.3 & 12.3 & 0.003 \\
\hline Live in elderly care facilities on admission (\%) & 7 & 45.6 & 47.4 & $<0.001$ \\
\hline MBI on admission & $49.9 \pm 18.6$ & $44.2 \pm 17.6$ & $34.2 \pm 18.2$ & $<0.001$ \\
\hline Length of stay (day) & $32.0 \pm 18.6$ & $37.1 \pm 47.8$ & $26.5 \pm 15.8$ & 0.648 \\
\hline In-patient mortality (\%) & 6.5 & 0.9 & 10.5 & 0.013 \\
\hline Six-month mortality (\%) & 8.7 & 8.7 & 19.3 & 0.097 \\
\hline
\end{tabular}

Values are presented as mean \pm standard deviation or number (\%).

CCI, Charlson Comorbidity Index; MMSE, Mini-Mental State Examination; MBI, Modified Barthel Index.

Statistical test: ANOVA for age, CCI, MMSE, albumin level, Hb level, length of stay, MBI on admission; chi-square test for sex, functional status, live in elderly care facilities on admission, in-patient and 6-month mortality. 
dent, 122 (56\%) walked with aids, and 12 (5.5\%) were either chair- or bed-bound. The majority of patients $(n=161$, $73.9 \%)$ were living at home. There were $102(46.8 \%)$ trochanteric fractures and 116 (53.2\%) neck fractures of the femur. Surgery was not done for 15 subjects, 89 (40.8\%) underwent proximal femoral nail antirotation, 50 (22\%) had a dynamic hip screw inserted, 61 (28\%) received hemiarthroplasty, and $3(1.4 \%)$ received Austin Moore arthroplasty. The average time from admission to operation was $3.2 \pm 7.9$ days and the average length of stay in the convalescence and rehabilitation unit was $33.2 \pm 36.8$ days. 136 (62.4\%) developed complications during the hospital stay. The complications included urinary tract infection $(n=29)$, acute retention of urine $(n=36)$, chest infection $(\mathrm{n}=35)$, delirium $(\mathrm{n}=15)$, and acute coronary syndrome $(n=5)$. The MNA-SF assessment indicated that 46 (21.1\%) patients were well nourished, 115 (52.6\%) were at risk of malnutrition, and 57 (26.1\%) were malnourished. There were $10(4.6 \%)$ in-hospital mortalities and a further 15 (11.5\%) had died by 6 months. MNA-SF significantly correlated with MBI gain on discharge $(\mathrm{r}=0.87, \mathrm{p}<0.001)$ and at 6 months $(\mathrm{r}=0.39, \mathrm{p}<0.001)$. The univariate analysis of clinical and functional outcomes among the three nutritional groups are presented in Table 1. Patients in the malnourished group were significantly older, had lower MMSE and albumin level, were more functionally dependent and were more likely to reside in an elderly care facility. Physical function was lower in the malnutri-

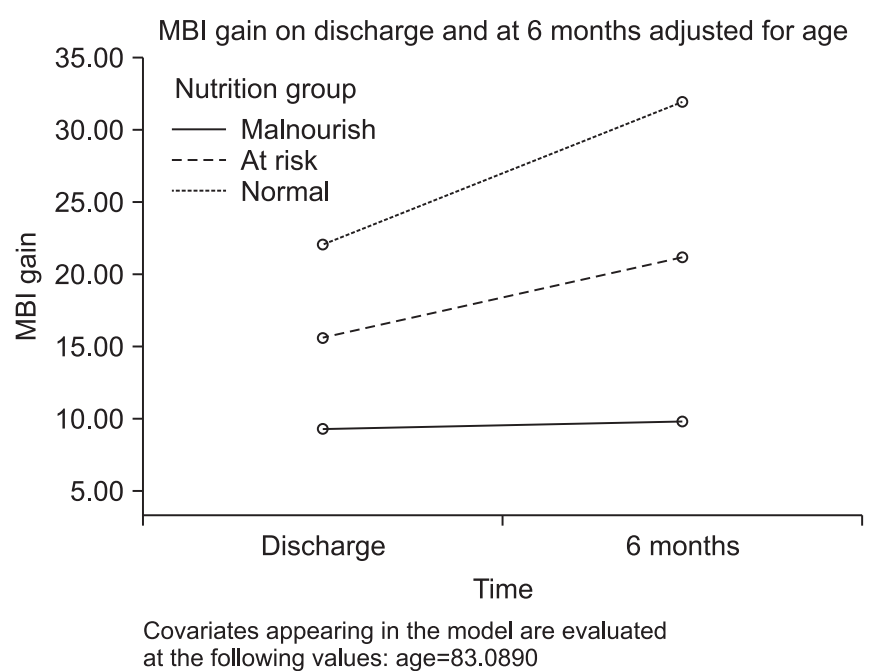

Fig. 1. Modified Barthel Index (MBI) change among nutritional group on discharge and at 6 months. Each line represent different nutritional group. tion group on admission. In-patient mortality rate was higher in the malnourished group than those at risk and in the well-nourished group. This pattern persisted after adjusting for age. At 6 months, there was no statistical significant difference of mortality rate between the three groups. Univariate analysis revealed that nutritional status, presence of post-operative complications, mobility status, age, hemoglobin and albumin level were associated with in-patient mortality. Logistic regression found that only albumin level (OR=0.86; 95\% CI, 0.77-0.97) was the independent predictor for in-patient death. For 6 months mortality, mobility status, age, MMSE, hemoglobin, albumin, MBI and BMI were significantly associated. The independent predictors for 6-month mortality after logistic regression analysis were $\mathrm{BMI}(\mathrm{OR}=0.811 ; 95 \% \mathrm{CI}$, 0.68-0.97), hemoglobin level (OR=0.62; 95\% CI, 0.47-0.81) and living in an elderly care facility at the time of admission (OR=4.22; 95\% CI, 1.12-15.9). Length of hospital stay showed no statistical significant difference. Fig. 1 displays the recovery rate among the nutritional groups. The recovery rate in activities of daily living (ADL) was slower in the malnutrition group even after adjustment for age, MMSE and place of residence on admission $(p<0.001)$ (Fig. 1). On discharge, the at-risk and the malnourished groups had a higher proportion of elderly care residents. The pattern persisted 6 months after discharge (Fig. 2).

\section{DISCUSSION}

The present study found a $26 \%$ prevalence of malnutrition among a cohort of elderly hip fracture patients. This

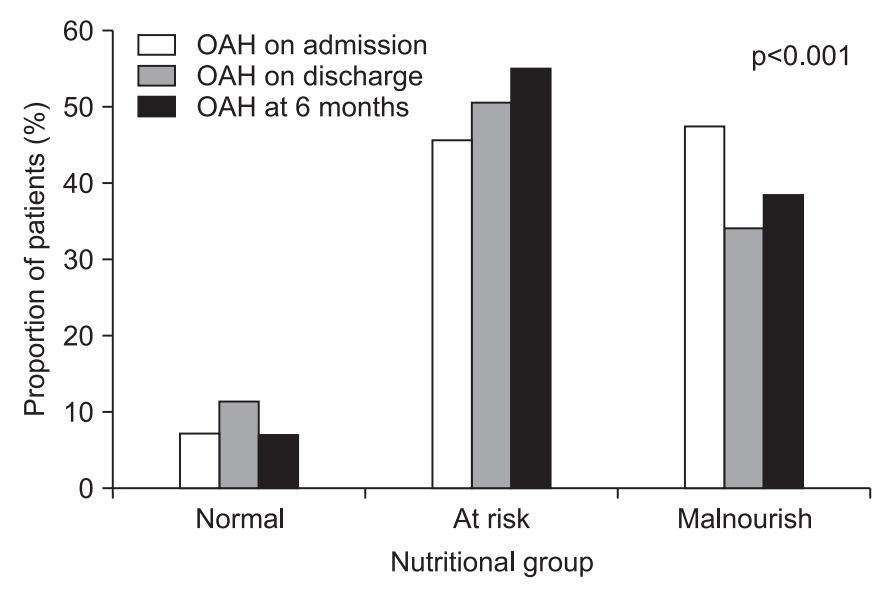

Fig. 2. Elderly care facilities (OAH) placement among nutritional group on admission, discharge and at 6 months. 
group of subjects was older, cognitively impaired and functionally more dependent.

There is no gold standard or consensus for diagnosis and documentation of protein energy malnutrition [17]. A study done among hip fracture in-patients [20] reported that the prevalence rate of malnutrition in a sample of acute hip fracture in-patients varied from 13\%-55\% depending on the diagnostic measurements used. Generally, BMI, albumin level, physician diagnosis and MNA are widely used. Presently, we used MNA-SF as the tool for screening of malnutrition. This assessment tool is recommended by international societies including the European Society for Enteral and Parenteral Nutrition (ESPEN), the International Association of Gerontology and Geriatrics (IAGG) and the International Academy for Nutrition in the Aged (IANA). Advantages of the MNA-SF for malnutrition diagnostic purposes include ease of application, minimal training and no need of biochemical measurements. MNA-SF has fair agreement with ICD10-AM and BMI diagnosis [20]. It is more likely to predict malnutrition-related outcomes than geriatrician diagnosis, BMI or albumin level.

The aim of nutritional assessment is to identify patients who are malnourished or at high risk of malnutrition. With appropriate nutritional intervention, it is hoped that they will benefit [21]. Failure to diagnose hospital-based malnutrition can lead to significant cost implications independent of whether nutritional intervention care will lead to an improvement in outcome [22].

The prevalence of malnutrition was high is our study. If we combined those who are at risk of malnutrition with the malnourished group by MNA-SF, nearly $80 \%$ of our study population belonged to this group. A prior study reported a $56 \%$ rate of undernourished and at risk of malnutrition patients with hip fracture upon admission [23]. On the other hand, a prospective cohort study [24] reported rates of $1.5 \%$ and $28 \%$ for malnourished and at risk of malnutrition among a cohort of hip fracture patients. However, many of the subjects had severe dementia and patients with pacemakers were excluded. Moreover, the mean age of the patients was considerably younger. The risk of malnutrition was higher when compared with the younger counterparts. This can be explained by the agerelated physiological, pathological, psychological and socio-economic changes [25].

Functional recovery is much lower among the mal- nourished and at risk groups. Presently, the admission MBI is lower in the malnourished and at risk groups. Those in the well-nourished group showed an improvement in MBI up to 3 times than that of the malnourished group on discharge (22.4 vs. 8.1) and at 6 months (32.7 vs. 9.3). Up to one-third of our study population were malnourished. Furthermore, the detrimental effects of preoperative fasting, injury-related inflammatory response and surgical stress may further increase catabolism [26]. We observed that the malnourished subjects had poor trajectories in performance of ADL and recovery of functional mobility than those without malnutrition. The this observation highlights the need to pay attention to the nutritional status of elderly hip fracture patients.

Not much information is available on the effect of nutritional status of older hip fracture patients prior to discharge. One study reported that the recovery rate of ADL and walking ability in the malnourished group was the worst, whereas the recovery of the group with interdisciplinary intervention including geriatric assessment and nutritional education was best at 3, 6, and 12 months after discharge [27]. Results concerning nutritional intervention are contradictory. Nutrition intervention reportedly improved the outcome of hip fracture patients in terms of reduction of long-term complications, hospital stay and mortality rate in one study [28]. But, energy supplementation did not show any improvement in functional recovery following hip fracture in another study [5]. It may be that the detrimental effect of malnutrition on skeletal muscle and organ system function requires a longer time to recover and the duration of energy supplementation should be prolonged beyond the hospital stay. The limited duration of energy supplement during the in-hospital stay period may not be sufficient to show a positive effect on functional outcome.

Many of the malnourished elderly hip fracture patients with poor functional recovery resided in elderly care facilities. Many failed to return home on discharge and at 6 months. An interaction between malnutrition and elderly care placement has been described. Residents residing in elderly care facilities have poorer nutritional status, which in turn leads to limited functional recovery. Generally, those who have poor premorbid health, multiple comorbidities and poor self-care ability are placed in elderly care facilities. Their functional reserves are already very low, which compromises functional recovery after 
hip fracture. Universal nutrition supplementation for all elderly care residents would have huge implications for the allocation of resources and funds. Health authorities should be involved in the introduction of dietary plans for elderly care residents at risk of malnutrition to reduce the comorbidities and promote functional recovery of these residents in case they are admitted to hospital. It is recommended that a detailed nutritional assessment and instruction program on nutritional care should be included in the early phase both before and in the immediate post-operative period, together with a home-based intervention program to minimize associated morbidity, mortality and improve functional recovery of elderly hip fracture patients, with the hope of reduced healthcare costs.

Presently, in-patient mortality was higher among the malnourished group but lowest in the at-risk group. On the other hand, the length of stay for this group is also highest, although it was not statistically significant. It could be that the at-risk group represents a group of patients with medical or social comorbidities that require longer hospital stay for intervention, and that medical and/or nutritional intervention may help to lower the mortality within this relatively longer hospital stay. Nonetheless, at 6 months post-discharge, there was no statistically significant difference in the mortality rate between the nutritional groups. In our study, after regression analysis, the only independent variable for inpatient mortality was albumin level, while hemoglobin level, BMI and living in an elderly care facility were independent predictors of 6 -month mortality. Generally, poor nutritional status was reflected by lower levels of albumin and hemoglobin, BMI was a poor prognostic marker for mortality. These observations highlight the limitation of applying MNA on mortality prediction. Lower nutritional status as assessed by MNA may be the result of a more profound process, such as a medical condition, depression, swallowing problem, normal ageing or mental and functional decline.

There are limitations in our study. Although patients identified as being malnourished were referred to a dietitian and managed with energy supplementation, reassessment data of their nutritional status on discharge are unavailable. It is not known whether their nutritional status improved during their stay in the hospital. Such a limited period of in-patient energy supplementation may not show any positive effect on physical functioning, morbidity and mortality.

There is a potential impact of under- or over-diagnosis of malnutrition, as other outcome measures such as utilization of healthcare resources were not collected. Further investigation is necessary to study the impact of malnutrition in the long term, especially for the allocation of resources. Nonetheless, this study is a representative sample of elderly hip fracture patients with high rate of cognitive impairment, functional limitation and high comorbid burden, which is commonly encountered in both acute and rehabilitation setting.

In conclusion, the prevalence of malnutrition and those at risk of malnutrition is high among a cohort of elderly hip fracture patients. It is associated with poor functional recovery and elderly care placement. Despite this, little nutritional screening is perform in orthopedic and surgical departments. Given the high prevalence of malnutrition and its impact on outcome, prevention and early intervention are essential. Daily protein and calorie supplement should be prescribed as it is easy to implement. Elderly care residents are an especially high-risk group since they have poorer premorbid functional status and have a higher proportion of malnourishment. Health authorities are encouraged to evaluate the dietetic component of elderly care and initiate nutrition supplementation in their planning of healthcare resources.

\section{CONFLICT OF INTEREST}

No potential conflict of interest relevant to this article was reported.

\section{ACKNOWLEDGMENTS}

This study is supported by Tung Wah Group of Hospitals (TWGHs) research fund.

\section{REFERENCES}

1. Miller CW. Survival and ambulation following hip fracture. J Bone Joint Surg Am 1978;60:930-4.

2. Magaziner J, Hawkes W, Hebel JR, Zimmerman SI, Fox KM, Dolan M, et al. Recovery from hip fracture in eight areas of function. J Gerontol A Biol Sci Med Sci 2000;55:M498-507. 
3. Shyu YI, Chen MC, Liang J, Wu CC, Su JY. Predictors of functional recovery for hip fractured elders during 12 months following hospital discharge: a prospective study on a Taiwanese sample. Osteoporos Int 2004;15: 475-82.

4. Charlton KE, Nichols C, Bowden S, Lambert K, Barone $\mathrm{L}$, Mason M, et al. Older rehabilitation patients are at high risk of malnutrition: evidence from a large Australian database. J Nutr Health Aging 2010;14:622-8.

5. Olofsson B, Stenvall M, Lundstrom M, Svensson O, Gustafson Y. Malnutrition in hip fracture patients: an intervention study. J Clin Nurs 2007;16:2027-38.

6. Chen CC, Schilling LS, Lyder CH. A concept analysis of malnutrition in the elderly. J Adv Nurs 2001;36:13142.

7. Allison SP. Malnutrition, disease, and outcome. Nutrition 2000;16:590-3.

8. Dawson-Hughes B. Interaction of dietary calcium and protein in bone health in humans. J Nutr 2003;133: 852S-4S.

9. Bonjour JP. Dietary protein: an essential nutrient for bone health. J Am Coll Nutr 2005;24(6 Suppl):526S$36 \mathrm{~S}$.

10. Paillaud E, Bories PN, Le Parco JC, Campillo B. Nutritional status and energy expenditure in elderly patients with recent hip fracture during a 2-month follow-up. Br J Nutr 2000;83:97-103.

11. Neumann M, Friedmann J, Roy MA, Jensen GL. Provision of high-protein supplement for patients recovering from hip fracture. Nutrition 2004;20:415-9.

12. Nikkel LE, Fox EJ, Black KP, Davis C, Andersen L, Hollenbeak CS. Impact of comorbidities on hospitalization costs following hip fracture. J Bone Joint Surg Am 2012;94:9-17.

13. Foss NB, Jensen PS, Kehlet H. Risk factors for insufficient perioperative oral nutrition after hip fracture surgery within a multi-modal rehabilitation programme. Age Ageing 2007;36:538-43.

14. Norman K, Pichard C, Lochs H, Pirlich M. Prognostic impact of disease-related malnutrition. Clin Nutr 2008;27:5-15.

15. Volkert D, Berner YN, Berry E, Cederholm T, Coti Bertrand P, Milne A, et al. ESPEN Guidelines on Enteral Nutrition: Geriatrics. Clin Nutr 2006;25:330-60.

16. Milte R, Miller M. Dietetic care of hip fracture patients across Australia: are we doing enough? Nutr Diet
2011;68:214-20.

17. White JV, Guenter P, Jensen G, Malone A, Schofield M, Academy Malnutrition Work Group, et al. Consensus statement: Academy of Nutrition and Dietetics and American Society for Parenteral and Enteral Nutrition: characteristics recommended for the identification and documentation of adult malnutrition (undernutrition). JPEN J Parenter Enteral Nutr 2012;36:275-83.

18. Kaiser MJ, Bauer JM, Ramsch C, Uter W, Guigoz Y, Cederholm T, et al. Validation of the Mini Nutritional Assessment short-form (MNA-SF): a practical tool for identification of nutritional status. J Nutr Health Aging 2009;13:782-8.

19. Kwok T, Whitelaw MN. The use of armspan in nutritional assessment of the elderly. J Am Geriatr Soc 1991;39:492-6.

20. Bell JJ, Bauer JD, Capra S, Pulle RC. Concurrent and predictive evaluation of malnutrition diagnostic measures in hip fracture inpatients: a diagnostic accuracy study. Eur J Clin Nutr 2014;68:358-62.

21. Velasco C, Garcia E, Rodriguez V, Frias L, Garriga R, Alvarez J, et al. Comparison of four nutritional screening tools to detect nutritional risk in hospitalized patients: a multicentre study. Eur J Clin Nutr 2011;65: 269-74.

22. Gout BS, Barker LA, Crowe TC. Malnutrition identification, diagnosis and dietetic referrals: are we doing a good enough job? Nutr Diet 2009;66:206-11.

23. Nematy $\mathrm{M}$, Hickson $\mathrm{M}$, Brynes $\mathrm{AE}$, Ruxton $\mathrm{CH}$, Frost GS. Vulnerable patients with a fractured neck of femur: nutritional status and support in hospital. J Hum Nutr Diet 2006;19:209-18.

24. Hoekstra JC, Goosen JH, de Wolf GS, Verheyen CC. Effectiveness of multidisciplinary nutritional care on nutritional intake, nutritional status and quality of life in patients with hip fractures: a controlled prospective cohort study. Clin Nutr 2011;30:455-61.

25. Koren-Hakim T, Weiss A, Hershkovitz A, Otzrateni I, Grosman B, Frishman S, et al. The relationship between nutritional status of hip fracture operated elderly patients and their functioning, comorbidity and outcome. Clin Nutr 2012;31:917-21.

26. Hedstrom M, Ljungqvist O, Cederholm T. Metabolism and catabolism in hip fracture patients: nutritional and anabolic intervention: a review. Acta Orthop 2006;77:741-7. 
27. Li HJ, Cheng HS, Liang J, Wu CC, Shyu YI. Functional recovery of older people with hip fracture: does malnutrition make a difference? J Adv Nurs 2013;69:1691703.
28. Milne AC, Potter J, Vivanti A, Avenell A. Protein and energy supplementation in elderly people at risk from malnutrition. Cochrane Database Syst Rev 2009;2: CD003288. 